\title{
APSS - Software Support for Decision Making in Statistical Process Control
}

\author{
DOI: 10.12776/QIP.V22I3.1141
}

\author{
Darja Noskievičová
}

Received: 01 August 2018 Accepted: 06 November 2018 Published: 31 November 2018

\begin{abstract}
Purpose: SPC can be defined as the problem solving process incorporating many separate decisions including selection of the control chart based on the verification of the data presumptions. There is no professional statistical software which enables to make such decisions in a complex way.

Methodology/Approach: There are many excellent professional statistical programs but without complex methodology for selection of the best control chart. Proposed program in Excel APSS (Analysis of the Process Statistical Stability) solves this problem and also offers additional learning functions.

Findings: The created SW enables to link altogether separate functions of selected professional statistical programs (data presumption verification, control charts construction and interpretation) and supports active learning in this field.

Research Limitation/implication: The proposed SW can be applied to control charts covered by SW Statgraphics Centurion and Minitab. But there is no problem to modify it for other professional statistical SW.

Originality/Value of paper: The paper prezents the original SW created in the frame of the research activities at the Department of Quality Management of FMT, VŠB-TUO, Czech Republic. SW enables to link altogether separate functions of the professional statistical SW needed for the complex realization of statitical process control and it is very strong tool for the active learning of statistical process control tasks.
\end{abstract}

Category: Research paper

Keywords: statistical process control; selection of control chart; shewhart charts; nonconventional control charts; active learning 


\section{INTRODUCTION}

Statistical process control (SPC) is an approach to process control that has been widely used in various industrial or non-industrial fields. SPC is based on so called Shewhart's conception of the process variability. This conception distinguishes variability caused by obviously effected common causes (process is considered to be statistically stable) from variability caused by abnormal assignable causes (process is considered to be out of control).

The main goal of SPC (Keller, 2011; Montgomery, 2012; Oakland, 2011; Qiu, 2014, Thompson and Koronacki, 2002) is an identification of abnormal variability caused by assignable causes with the aim to:

- make the process stable,

- minimize the process variability,

- improve the process performance.

The application of SPC must be built as the problem-solving process (Noskievičová, 2010). By the SPC design the general structure of the problemsolving process must be respected and the sequence of the subprocesses "Out-of control signal revelation - Root cause identification - Corrective or improvement action acceptance - verification of action" must be the axis of every SPC application.

SPC as the problem solving process incorporate the decision making processes i.e. many decisions about various statistical, methodological, social and economical factors affecting the efficiency of the SPC (Noskievičová, 2010). Decisions linked to the main goals of SPC refer to the out-of control signal revelation (1), identification of root cause of assignable causes (2), selection of corrective or improvement action (3) and their realization (4). There are many excellent accessible professional statistical programs such as Statistica (Dell Statistica Help, 2017); Statgraphics (Statgraphics Technologies, 2017); Minitab (Minitab, 2018) etc. But there is no SW support for very important decision which precedes activities (1) - (4): selection of the suitable type of the control chart (which is one of the most important activities influencing the efficiency of the whole SPC implementation) based on the properly made verification of statistical properties of data (Cox, 2006; Zimmerman, 2011). Above mentioned statistical software offer various types of control charts, various methods for data verification but there is no complex methodology how to select the best control chart.

For that reason the SW program in Excel called APSS (Analysis of the Process Statistical Stability), solving this failure of the professional statistical packages with additional learning functions was created at the Department of Quality Management of the Faculty of Metallurgy and Material Engineering, VŠBTechnical University of Ostrava, Czech Republic. The paper deals with goals, principles, functions and a structure of this program. 


\section{GOALS, PRINCIPLES AND FUNCTIONS OF APSS}

\subsection{Goals}

The main goals of APSS are as follows (Noskievičová, 2015):

- Integration of partial steps of SPC into one complex solution;

- Support of learning of the decision-making activities in the frame of the verification and ensuring the statistical stability of the processes, i.e. by the selection of the suitable control charts and by the interpretation of the reached results;

- Increasing quality of the self-learning process.

\subsection{Principles and Functions}

APSS enables to realize activities (see Fig. 1) in one complex environment at all main connections. Except the application of particular control charts users will learn to select suitable control chart based on the statistical data analysis and to interpret correctly reached results.

APSS guarantees following functions: selfstudy, communication, decisionsupport, control, verification of obtained knowledge, data support, methodical support.

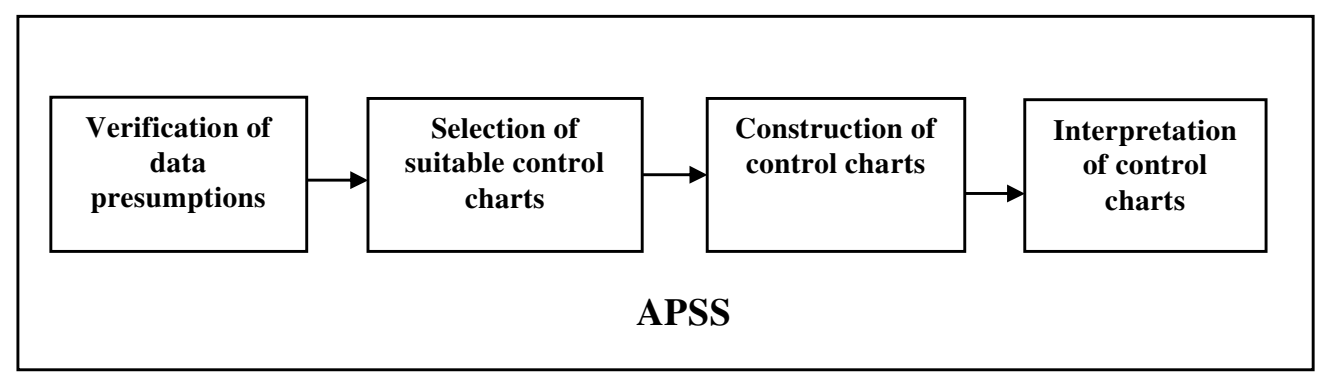

Figure 1 - The Basic Tasks of APSS (Source: Author's Elaboration)

\section{APSS STRUCTURE}

The basic structure of APSS with its basic elements and outer and inner relations is depicted on the Fig. 2. APSS itself forms the environment for practicing intended tasks, decision-making, verification of these decisions (testing questions and evaluation of the results), quick complementing missing knowledge (elearning study materials written by the author of this paper concerning the problems of classical Shewhart and nonconventional control charts) and communication between user and professional statistical SW (APSS supposes that users are able to use professional statistical SW Statgraphics and Minitab). This professional statistical SW is used for the construction of control charts 
selected by user, some activities connected to the control chart analysis and verification of data presumptions.

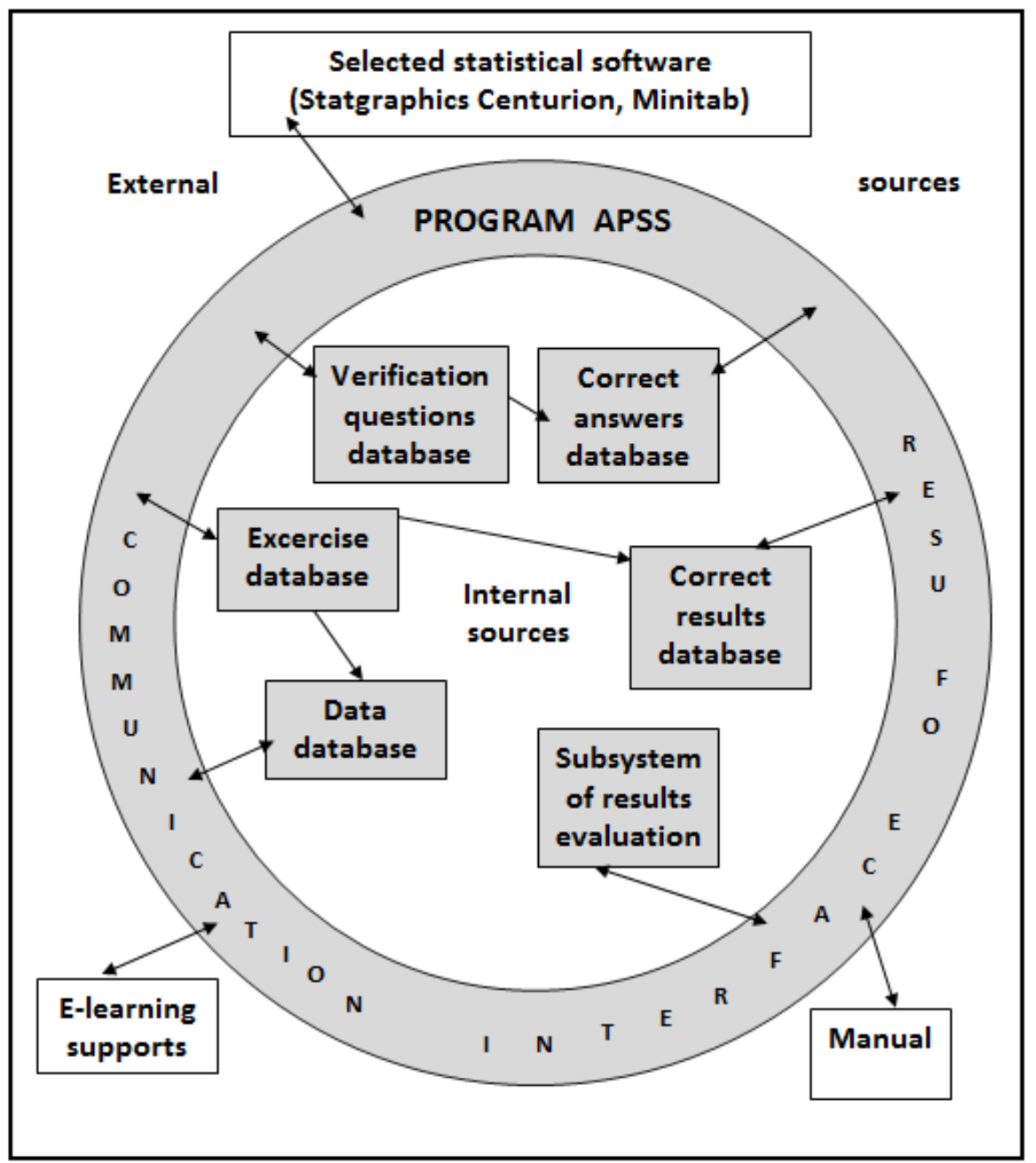

Figure 2 - Structure of APSS and its Basic outer and Inner Relations (Source: Author's Elaboration)

APSS has been created as an opened system. It means that all databases can be complemented and whatever statistical SW can be used (but under the condition of adequate changes in the exercises and database of correct results).

More detailed information about the structure, functioning and contents of APSS is given by Fig. 3 . 


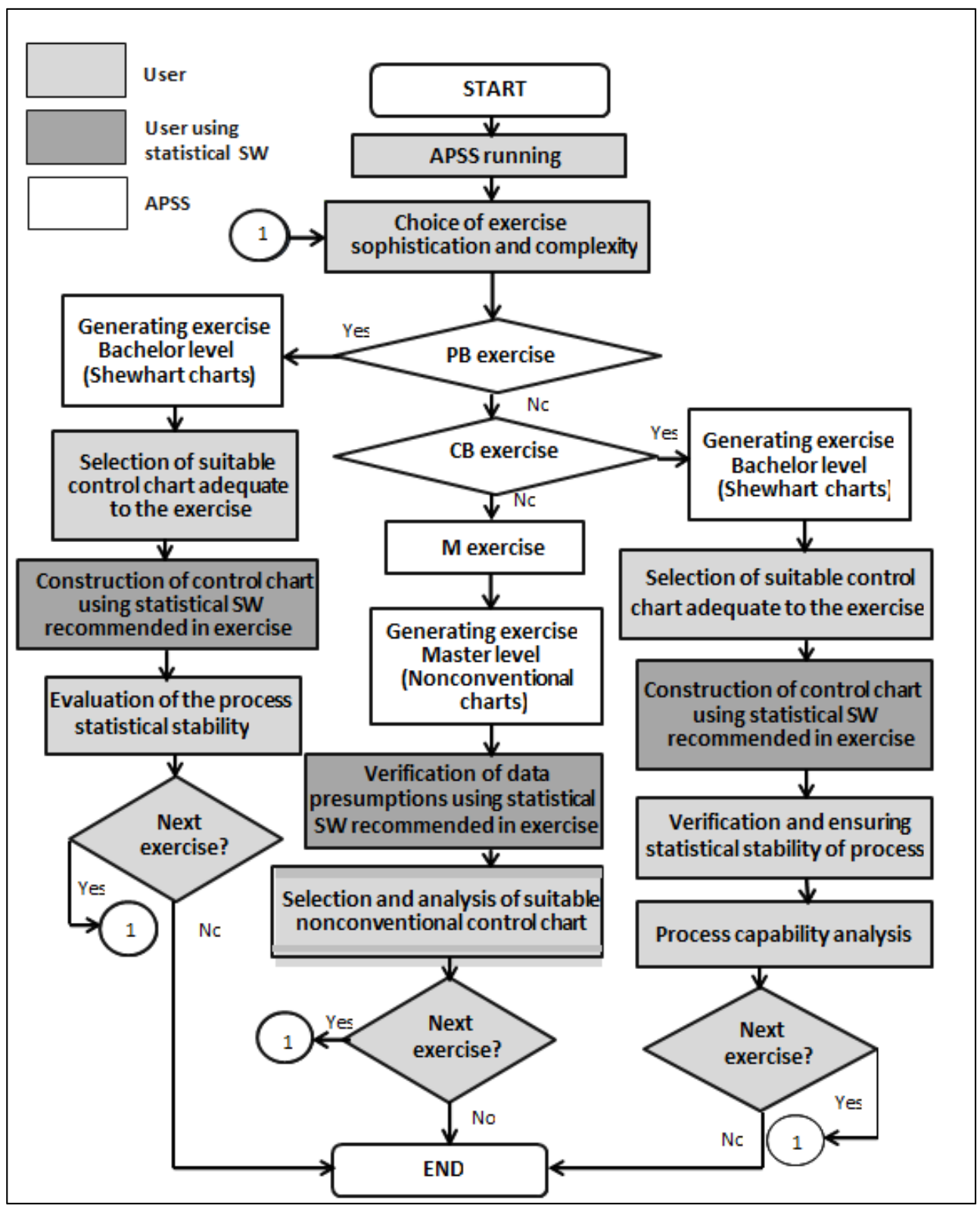

Figure 3 - Flow Chart of the Detailed APSS Structure

(Source: Author's Elaboration)

Steps in white blocks are done in the frame of APSS, the steps in grey ones are realized using the recommended professional statistical SW.

APSS is divided into three main modules according to the sophistication and complexity of exercises. The first module is dedicated to the solution of particular bachelor (PB) exercises - it covers selection and analysis of Shewhart conventional control charts. The second module corresponds to the bachelor level 
of knowledge of SPC and it is dedicated to the solution of complex bachelor (CB) exercises - in addition to the previous module it contains also process capability analysis. The third module corresponds to our master level of knowledge of SPC (M exercises). It covers selection of suitable nonconventional control chart and its analysis when data presumptions for the application of Shewhart conventional control charts are not met. This module also contains verification of these data presumptions. All modules have the same feature from every communication screen of the program the user is able to the solved exercise, the exercise data or to the manual.

In the next paragraphs there is more detailed description of these modules.

\subsection{Module of PB Exercises}

This module starts with automatic generating exercise. Then the selection of suitable control chart is done. When the selection is not correct the user is recommended to move to the e-learning texts which are linked to APSS and to study the problem. Then the verification test in APSS is activated. The user must reach 10 points otherwise he must repeat the whole test with upgraded questions. When the selection of control chart is correct the parameters of control chart (subgroup size $n$ and number of subgroups $k$ ) are entered. If these values are not correct the user is recommended to go back to the exercise and study it carefully again. Having correct parameters $n$ and $k$ the user must open SW recommended in the exercise, copy the data from the exercise into it and to construct previously selected control charts. User's results are then compared to the correct solution in APSS. When there is no conformity the user is recommended to move to the elearning texts and to study the problem. In the case of the conformity APSS continues by the verification of the user knowledge about statistical stability analysis. When his answers are not correct he is recommended to move to the elearning texts and to study the problem. When answers are correct the user must move to the applied statistical SW and to verify statistical stability of the process from the solved exercise. His results are again compared to the correct solution. After it there are some questions about the following steps of the SPC. In the case of incorrect answers there is again need to study the problem using the linked study texts. Otherwise the user can choose the next exercise or to finish.

\subsection{Module of CB Exercises}

This module contains the whole previous module and after verification of statistical stability it asks the questions about capability analysis. When answer is not correct the user must move to e-learning material. Then he must go to the opened statistical SW and to recompute control limits of the previously constructed and analysed control chart. Then the estimated values of the process parameters needed for the capability indices computations are entered into APSS. Again APSS compares the user results with the correct solution. When there is no conformity the user must go back to the setting the estimations of the process 
parameters and again to compute capability indices. Otherwise he can continue with the interpretation these results. When his conclusions are not correct the user is recommended to go to the linked e-learning materials and to study the problem. In the case of the correct interpretation the user can choose the next exercise or to finish.

\subsection{Module of M Exercises}

This module teaches the user to verify data presumptions using suitable statistical tests and to select suitable control charts based on this statistical analysis. It contains 5 branches. The first branch enables solution when data are not normally distributed. The second branch gives solution in the case of data autocorrelation; in the third branch the user learns to solve the situation when little changes of the process parameters must be identified; the fourth branch is dedicated to the problem of the lack of the data about the process and the last branch offers solution when more then one quality characteristic must be watched simultaneously.

\section{CONCLUSION}

This paper deals with the description of the software product which was worked out for the practicing selection of the suitable control chart based on verification of the data. In the first chapter SPC is defined as the problem solving incorporating many decisions including also selection of the suitable control chart which must be based on the verification of the data presumptions. The second chapter is dedicated to the description of the goals, principles and functions of APSS (Analysis of the Process Statistical Stability), SW program programed in Excel, that was created in the Department of Quality Management at the Faculty of Metallurgy and Material Engineering, VŠB - Technical University of Ostrava, Czech Republic to support correct selection of control charts. In the third chapter there is general description of the whole program and also more detailed characterization of its parts. The program is very useful instrument for learning how to correctly choose suitable control chart based on the verification of the data properties.

\section{ACKNOWLEDGEMENTS}

This paper was elaborated in the frame of the specific research project SP2018/109 which has been solved at the Faculty of Metallurgy and Materials Engineering, VŠB - TU Ostrava with the support of Ministry of Education, Youth and Sports, Czech Republic. 


\section{REFERENCES}

Cox, D.R., 2006. Principles of Statistical Inference. Cambridge: Cambridge University Press.

Dell Statistica Help, 2017. Help. [online] Available at: $<$ http://documentation.statsoft.com/STATISTICAHelp.aspx?path=common/Abo utSTATISTICA/ElectronicManualIndex> [Accessed 01 August 2018].

Keller, P.A., 2011. Statistical Process Control Demystified. New York: McGrawHill.

Minitab, 2018. Minitab. [online] Available at: <http://www.minitab.com/enus/products/minitab/> [Accessed 01 August 2018].

Montgomery, D.C., 2013. Statistical Quality Control: A Modern Introduction. $7^{\text {th }}$ Ed. Hoboken: J. Wiley \& Sons.

Noskievičová, D., 2010. Effective Implementation of Statistical Process Control, Engineering the Future. In: L. Dudas, ed. 2010. Engineering the Future. London: InteChopen. Ch. 11.

Noskievičová, D., 2015. Module 4 Process Improvement Using Statistical Analysis, Submodule 4.4 Analysis of the Process Statistical Stability, User manual. Ostrava: VŠB-TU Ostrava.

Oakland, J.S., 2011. Statistical Process Control. $6^{\text {th }}$ Ed. New York: Routledge.

Qiu, P., 2014. Introduction to Statistical Process Control. Boca Raton: CRC Press.

Statgraphics Technologies, 2017. Statgraphics 18. [online] Available at: $<$ http://www.statgraphics.com/centurion-xvii > [Accessed 01 August 2018].

Thompson, J.R. and Koronacki, J., 2002. Statistical Process Control: the Deming Paradigm and Beyond. $2^{\text {nd }}$ ed. Boca Raton: Chapman \& Hall/CRC.

Zimmerman, D.W., 2011. A Simple and Effective Decision Rule for Choosing a Significance Test to Protect Against Non-normality. Br. J. Math. Stat. Psychol., 64(3), pp.388-409. http://dx.doi.org/10.1348/000711010X524739.

\section{ABOUT THE AUTHOR}

Darja Noskievičová - prof. Ing. CSc., Department of Quality Management, Faculty of Metallurgy and Materials Engineering, VŠB - Technical University of Ostrava, 17. listopadu 15, 70833 Ostrava-Poruba, Czech Republic. E-mail: darja.noskievicova@vsb.cz.

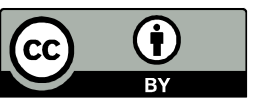

(C) 2018 by the authors. Submitted for possible open access publication under the terms and conditions of the Creative Commons Attribution (CC-BY) license (http://creativecommons.org/licenses/by/4.0/). 